\title{
INTRINSIC ULTRACONTRACTIVITY FOR GENERAL LÉVY PROCESSES ON BOUNDED OPEN SETS
}

\author{
XIN CHEN JIAN WANG
}

\begin{abstract}
We prove that a general (not necessarily symmetric) Lévy process killed on exiting a bounded open set (without regular condition on the boundary) is intrinsically ultracontractive, provided that $B\left(0, R_{0}\right) \subseteq \operatorname{supp}(\nu)$ for some constant $R_{0}>0$, where $\operatorname{supp}(\nu)$ denotes the support of the associated Lévy measure $\nu$. For a symmetric Lévy process killed on exiting a bounded Hölder domain of order 0 , we also obtain the intrinsic ultracontractivity under much weaker assumption on the associated Lévy measure.
\end{abstract}

Keywords: Lévy process; Dirichlet semigroup; intrinsic ultracontractivity; super Poincaré inequality

MSC 2010: 60G51; 60G52; 60J25; 60J75.

\section{Introduction and Main Results}

\subsection{Dirichlet Semigroup and its Dual Semigroup for General Lévy Pro-} cess. Let $X=\left(\left(X_{t}\right)_{t \geqslant 0}, \mathbb{P}^{x}\right)$ be a Lévy process on $\mathbb{R}^{d}$ with Lévy triplet $(Q, b, \nu)$, such that its characteristic exponent is given by

$$
q(\xi)=\frac{1}{2}\langle\xi, Q \xi\rangle+i\langle\xi, b\rangle+\int_{\mathbb{R}^{d} \backslash\{0\}}\left(1-e^{i\langle\xi, z\rangle}+i\langle\xi, z\rangle \mathbb{1}_{\{|z| \leqslant 1\}}\right) \nu(d z), \quad \xi \in \mathbb{R}^{d},
$$

where $Q: \mathbb{R}^{d} \rightarrow \mathbb{R}^{d}$ is a symmetric non-negative definite $d \times d$ matrix, $b \in \mathbb{R}^{d}$, and $\nu$ is a Lévy measure on $\mathbb{R}^{d}$. Let $\hat{X}=\left(\hat{X}_{t}\right)_{t \geqslant 0}$ denote the dual process of $X$, which is a Lévy process with the Lévy triplet $(Q,-b, \hat{\nu})$ such that $\hat{\nu}(U)=\nu(-U)$ for any $U \in \mathscr{B}\left(\mathbb{R}^{d}\right)$. Throughout this paper, we assume that the process $X$ has a continuous, bounded and strictly positive transition density $p(t, x, y)=p(t, 0, y-x)$, i.e. for every $t>0$ and $f \in B_{b}\left(\mathbb{R}^{d}\right)$, (here and in what follows, $B_{b}\left(\mathbb{R}^{d}\right)$ denotes the set of bounded measurable functions on $\mathbb{R}^{d}$,)

$$
\mathbb{E}^{x} f\left(X_{t}\right)=\int_{\mathbb{R}^{d}} p(t, x, y) f(y) d y, \quad x \in \mathbb{R}^{d},
$$

$p(t, \cdot, \cdot): \mathbb{R}^{d} \times \mathbb{R}^{d} \mapsto(0, \infty)$ is continuous, and there is a constant $c(t)>0$ such that

$$
0<p(t, x, y) \leqslant c(t), \quad \forall x, y \in \mathbb{R}^{d} .
$$

See $[3,18,22,23,29,31]$ for sufficient conditions in terms of characteristic exponent $q(\xi)$.

Let

$$
T_{t} f(x)=\mathbb{E}^{x} f\left(X_{t}\right), \quad \hat{T}_{t} f(x)=\mathbb{E}^{x} f\left(\hat{X}_{t}\right) .
$$

X. Chen: Department of Mathematics, Shanghai Jiao Tong University, 200240 Shanghai, P.R. China. chenxin_217@hotmail.com.

J. Wang: School of Mathematics and Computer Science, Fujian Normal University, 350007 Fuzhou, P.R. China. jianwang@fjnu.edu.cn. 
Then for any non-negative Borel measurable function $f$ and $g$,

$$
\int T_{t} f(x) g(x) d x=\int f(x) \hat{T}_{t} g(x) d x .
$$

Hence, the (dual) Lévy process $\hat{X}$ also possesses a continuous, bounded and strictly positive transition density $\hat{p}(t, x, y)$ such that for any $t>0$ and $x, y \in \mathbb{R}^{d}, \hat{p}(t, x, y)=$ $p(t, y, x)$ and

$$
\mathbb{E}^{x} f\left(\hat{X}_{t}\right)=\int_{\mathbb{R}^{d}} \hat{p}(t, x, y) f(y) d y=\int_{\mathbb{R}^{d}} p(t, y, x) f(y) d y, \quad x \in \mathbb{R}^{d}, f \in \mathscr{B}_{b}\left(\mathbb{R}^{d}\right) .
$$

Let $D \subseteq \mathbb{R}^{d}$ be an open set. Define the following subprocess of $X$

$$
X_{t}^{D}:=\left\{\begin{array}{cl}
X_{t}, & \text { if } t<\tau_{D} \\
\partial, & \text { if } t \geqslant \tau_{D},
\end{array}\right.
$$

where $\tau_{D}:=\inf \left\{t>0: X_{t} \notin D\right\}$ and $\partial$ denotes the cemetery point. Then, the process $X^{D}:=\left(X_{t}^{D}\right)_{t \geqslant 0}$ is called the killed process of $X$ on exiting $D$. By the strong Markov property and the continuity of $p(t, \cdot, \cdot)$ for all $t>0$, it is easy to see that the process $X^{D}$ has a transition density (or Dirichlet heat kernel) $p^{D}(t, x, y)$, which enjoys the following relation with $p(t, x, y)$ :

$$
\begin{aligned}
& p^{D}(t, x, y)=p(t, x, y)-\mathbb{E}^{x}\left[p\left(t-\tau_{D}, X_{\tau_{D}}, y\right) \mathbb{1}_{\left\{t \geqslant \tau_{D}\right\}}\right], \quad x, y \in D ; \\
& p^{D}(t, x, y)=0, \quad x \notin D \text { or } y \notin D .
\end{aligned}
$$

According to (1.3), one can show that $p^{D}(t, x, y), t>0$, satisfy the ChapmanKolmogorov equation; moreover, for every $t>0$ the function $p^{D}(t, \cdot, \cdot): D \times D \mapsto$ $[0, \infty)$ is continuous, and $\sup _{x, y \in D} p^{D}(t, x, y) \leqslant \sup _{x, y \in \mathbb{R}^{d}} p(t, x, y)<\infty$, see e.g. the proof of $[12$, Theorem 2.4]. Define

$$
T_{t}^{D} f(x)=\mathbb{E}^{x} f\left(X_{t}^{D}\right)=\int_{D} p^{D}(t, x, y) f(y) d y, \quad t>0, x \in D, f \in L^{2}(D ; d x) .
$$

It is a standard result that $\left(T_{t}^{D}\right)_{t \geqslant 0}$ is a strongly continuous contraction semigroup on $L^{2}(D ; d x)$, which is called the Dirichlet semigroup associated with the process $X^{D}$. We further assume that $p^{D}(t, x, y)>0$ for every $t>0$ and $x, y \in D$, which is equivalent to saying that $\left(T_{t}^{D}\right)_{t \geqslant 0}$ is irreducible, i.e. $T_{t}^{D}\left(\mathbb{1}_{U}\right)(x)>0$ for every $t>0$, $x \in D$ and open set $U \subseteq D$ with $|U|>0$, where $|U|$ denotes the Lebesgue measure of $U$. We should mention that even if the transition density $p(t, x, y)$ is smooth and strictly positive, it is non-trivial to show the strict positivity of $p^{D}(t, x, y)$, see Proposition 2.3 below for some mild assumption on the Lévy measure.

Let $\hat{\tau}_{D}:=\inf \left\{t>0: \hat{X}_{t} \notin D\right\}$ be the first exit time from $D$ for the dual process $\hat{X}$. Similar to (1.2), we can define the killed process $\hat{X}^{D}:=\left(\hat{X}_{t}^{D}\right)_{t \geqslant 0}$ of $\hat{X}$ on exiting $D$. For any $t>0$ and $x \in D$, define

$$
\hat{T}_{t}^{D} f(x)=\mathbb{E}^{x} f\left(\hat{X}_{t}^{D}\right) .
$$

Due to Hunt's switching identity (see [1, Chapter II, Theorem 5]),

$$
\int_{D} f(x) T_{t}^{D} g(x) d x=\int_{D} g(x) \hat{T}_{t}^{D} f(x) d x
$$


Then, the killed process $\hat{X}^{D}$ also has a transition density $\hat{p}^{D}(t, x, y)$ such that $\hat{p}^{D}(t, x, y)=p^{D}(t, y, x)$ for all $t>0$ and $x, y \in D$, and so

$$
\hat{T}_{t}^{D} f(x)=\int_{D} p^{D}(t, y, x) f(y) d y, \quad t>0, x \in D, f \in L^{2}(D ; d x) .
$$

When the Lévy process $X$ is symmetric, the associated Lévy measure $\nu$ is symmetric, and the characteristic exponent $q(\xi)$ given by (1.1) is reduced into

$$
q(\xi)=\frac{1}{2}\langle\xi, Q \xi\rangle+\int(1-\cos \langle\xi, z\rangle) \nu(d z) .
$$

Then, $\left(T_{t}\right)_{t \geqslant 0}$ and $\left(T_{t}^{D}\right)_{t \geqslant 0}$ are symmetric semigroups on $L^{2}\left(\mathbb{R}^{d} ; d x\right)$ and $L^{2}(D ; d x)$, respectively. In particular, $T_{t}=\hat{T}_{t}$ and $T_{t}^{D}=\hat{T}_{t}^{D}$ for any $t>0$, and $p^{D}(t, x, y)=$ $\hat{p}^{D}(t, x, y)$ for any $t>0$ and $x, y \in D$.

1.2. Main Result. In this part, we always assume that $D$ is a bounded open subset of $\mathbb{R}^{d}$. Since $\sup _{x, y \in D} p^{D}(t, x, y)<\infty$ and $D$ is bounded, both $T_{t}^{D}$ and $\hat{T}_{t}^{D}$ are Hilbert-Schmidt operators on $L^{2}(D ; d x)$ for every $t>0$, and so they are compact. Noticing that $p^{D}(t, x, y)>0$ for all $x, y \in D$, it follows form Jentzsch's Theorem (see $\left[28\right.$, Chapter V, Theorem 6.6]) that the common value $-\lambda_{1}=\sup \operatorname{Re}\left(\sigma\left(L_{D}\right)\right)=$ $\sup \operatorname{Re}\left(\sigma\left(\hat{L}_{D}\right)\right)<0^{1}$ is an eigenvalue of multiplicity 1 for the operators $L_{D}$ and $\hat{L}_{D}$, which are $L^{2}(D ; d x)$-generators of $\left(T_{t}^{D}\right)_{t \geqslant 0}$ and $\left(\hat{T}_{t}^{D}\right)_{t \geqslant 0}$ respectively. Moreover, according to [21, Proposition 3.8], the corresponding eigenfunctions $\phi_{1}$ and $\hat{\phi}_{1}$ can be chosen to be bounded, continuous and strictly positive on $D$. In the literature, this eigenfunction $\phi_{1}$ (resp. $\hat{\phi}_{1}$ ) is named ground state (resp. dual ground state). We are interested in the intrinsic ultracontractivity of $\left(T_{t}^{D}\right)_{t \geqslant 0}$, which is defined that for every $t>0$, there exists a constant $C(t)>0$ such that

$$
p^{D}(t, x, y) \leqslant C(t) \phi_{1}(x) \hat{\phi}_{1}(y), \quad x, y \in D .
$$

The notion of intrinsic ultracontractivity for symmetric semigroups was first introduced by Davies and Simon in [14] (note that in symmetric setting, $\phi_{1}=\hat{\phi}_{1}$ in (1.4)), and then it was generalized to non-symmetric semigroups by Kim and Song in [19]. It has wide applications in the area of analysis and probability. Recently, the intrinsic ultracontractivity of Markov semigroups (including Dirichlet semigroups and Feyman-Kac semigroups) has been intensively established for various Lévy processes

\footnotetext{
${ }^{1}$ For any $f, g \in C_{c}^{\infty}(D ; d x)$,

$$
\int_{D} f(x) L_{D} g(x) d x=\int_{D} g(x) \hat{L}_{D} f(x) d x
$$
}

Since the associated Lévy measure $\hat{\nu}$ of $\hat{L}_{D}$ satisfies that $\hat{\nu}(U)=\nu(-U)$ for any $U \in \mathscr{B}\left(\mathbb{R}^{d}\right)$, we have for $f \in C_{c}^{\infty}(D ; d x)$

$$
\begin{aligned}
-\int_{D} f(x) L_{D} f(x) d x & =-\frac{1}{2} \int_{D} f(x)\left(L_{D}+\hat{L}_{D}\right) f(x) d x \\
& =\frac{1}{4} \int_{\mathbb{R}^{d}} \int_{\mathbb{R}^{d}}(f(x+z)-f(x))^{2}(\nu(d z)+\hat{\nu}(d z)) d x .
\end{aligned}
$$

Now, let $\phi_{1}$ be the normalized non-zero eigenfunction associated with $\lambda_{1}$. Since $L_{D} \phi_{1}=-\lambda_{1} \phi_{1}$ and $\phi_{1} \equiv 0$ on $D^{c}$, by the standard approximation,

$$
\lambda_{1}=-\int_{D} \phi_{1}(x) L_{D} \phi_{1}(x) d x=\frac{1}{4} \int_{\mathbb{R}^{d}} \int_{\mathbb{R}^{d}}\left(\phi_{1}(x+z)-\phi_{1}(x)\right)^{2}(\nu(d z)+\hat{\nu}(d z)) d x>0 ;
$$

otherwise $\phi_{1}$ is a constant function on $\mathbb{R}^{d}$, which is impossible. 
or Lévy type processes, see e.g. $[4,5,9,10,15,16,17,19,20,21,24,25,26]$. The aim of this paper is to study the intrinsic ultracontractivity of Dirichlet semigroup $\left(T_{t}^{D}\right)_{t \geqslant 0}$ for a discontinuous (not necessarily symmetric) Lévy process (which may contain Brownian motion) on a bounded open set $D$ with very mild conditions on its Lévy measures $\nu$ and the set $D$.

To state our first contribution, we need the following additional assumption on the Lévy measure $\nu$.

(A1) There exists a constant $R_{0}>0$ such that

$$
B\left(0, R_{0}\right) \subseteq \operatorname{supp}(\nu)
$$

where $B(x, r)$ denotes the ball with center $x \in \mathbb{R}^{d}$ and radius $r>0$, and $\operatorname{supp}(\nu)$ denotes the support of the Lévy measure $\nu$.

Note that for Lévy process with finite range jumps, the distance between connected components of $D$ should not be too far away, otherwise $p^{D}(t, x, y)$ will be zero there. Therefore, to ensure the strictly positivity of $p^{D}(t, x, y)$, we need the following roughly connected assumption on the open set $D$, e.g. see [21, Definition $4.3]$.

(RC) For any $x, y \in D$, there exist distinct connected components $\left\{D_{i}\right\}_{i=1}^{m}$ of $D$, such that $x \in D_{1}, y \in D_{m}$ and for every $1 \leqslant i \leqslant m-1$, $\operatorname{dist}\left(D_{i}, D_{i+1}\right)<R_{0}$, where $R_{0}$ is the constant in Assumption (A1).

Theorem 1.1. Let $X$ be the Lévy process as above such that assumption (A1) holds, and suppose that the open set D satisfies (RC). Then the associated Dirichlet semigroup $\left(T_{t}^{D}\right)_{t \geqslant 0}$ is intrinsically ultracontractive. More explicitly, there is a constant $c>0$ such that for all $t>0$ and $x, y \in D$,

$$
p^{D}(t, x, y) \leqslant \frac{c e^{-\lambda_{1} t}}{(t \wedge 1)^{2}}\left(\int e^{-(t \wedge 1) \operatorname{Re} q(\xi)} d \xi\right) \phi_{1}(x) \hat{\phi}_{1}(y),
$$

where $q(\xi)$ is the characteristic exponent of the process $X$ given by (1.1), and $-\lambda_{1}<$ 0 is the common eigenvalue corresponding to ground state $\phi_{1}$ and $\hat{\phi}_{1}$.

For symmetric Lévy process, [15] has established the intrinsic ultracontractivity of Dirichlet semigroup on any bounded open set $D$, when the associated Lévy measure has full support (i.e. (1.5) holds with $R_{0}=\infty$ ). For general Lévy process, if the Lebesgue measure is absolutely continuous with respect to Lévy measure, the intrinsic ultracontractivity of Dirichlet semigroup on any bounded open set $D$ was verified in [21]. Note that in the latter setting the associated Lévy measure also has full support, and the corresponding Lévy process has full range jumps. The reader can refer to [15] for other non-degenerate conditions on Lévy measure in the symmetric setting. On the other hand, when Lévy measure is compactly supported and the Radon-Nikodym derivative of absolutely continuous part of Lévy measure is bounded below by some positive constant near the origin, Kim and Song proved in [21] that the corresponding Dirichlet semigroup is intrinsically ultracontractive for general (not necessarily symmetric) Lévy process provided that $D$ is $\kappa$-fat, see [21, Assumption A4(b)]. The reader can also refer to [11] for the intrinsic ultracontractivity for the Dirichlet semigroup associated with a Brownian motion on different non-smooth domains. 
The new point of Theorem 1.1 is due to that, it gets rid of any regularity condition on bounded open set $D$ to ensure the intrinsic ultracontractivity of associated Dirichlet semigroups for general Lévy process with finite range jumps. Besides, we do not require that Lévy measure has an absolutely continuous part. See Example 3.4 in the end of Section 3 for an application of Theorem 1.1.

1.3. Symmetric Lévy Process on Bounded Hölder Domain of Order 0. Throughout the paper, we always refer to a connected open set as a domain. It is known that the intrinsic ultracontractivity of Dirichlet semigroups for Brownian motion on a bounded domain $D$ depends on the geometry of the boundary of $D$ (see [11]). Theorem 1.1 indicates that for Lévy process even with finite range jumps, the associated Dirichlet semigroup can be intrinsically ultracontractive without any regularity condition on the bounded domain. In fact, for more general bounded domains including bounded Hölder domain of order 0 , we can prove the intrinsic ultracontractivity of the associated Dirichlet semigroups for symmetric Lévy process, whose Lévy measure satisfies weaker assumption than (A1).

To be more explicit, we introduce the following logarithmic distance integrability assumption on the domain $D$.

(LDI) For each $\theta>0$,

$$
\int_{D}\left|\log \left(\frac{1}{\rho_{\partial D}(x)}\right)\right|^{\theta} d x<\infty
$$

where $\rho_{\partial D}(x)=\inf \{|x-y|: y \in \partial D\}$ denotes the distance between $x$ and the boundary of $D$.

According to the proof of [30, Theorem 2], any Hölder domain of order 0 satisfies (LDI). Note that, it is shown in [30] that a Hölder domain of order 0 is bounded. John domains, in particular bounded Lipschitz domains, are Hölder domains of order 0 . In fact, recall that a domain $D$ is called Hölder domain of order 0 if there exist some constants $c_{1}, c_{2}>0$ and $x_{0} \in D$, such that

$$
k_{D}\left(x_{0}, x\right) \leqslant c_{1} \log \left(\frac{1}{\rho_{\partial D}(x)}\right)+c_{2}, \quad \forall x \in D .
$$

Here, $k_{D}(x, y)$ is the hyperbolic distance between $x, y \in D$ defined by

$$
k_{D}(x, y):=\inf _{\gamma} \int_{0}^{1} \frac{|\dot{\gamma}(s)|}{\rho_{\partial D}(\gamma(s))} d s,
$$

where the infimum is taken over all the rectifiable curves $\gamma:[0,1] \rightarrow D$ such that $\gamma(0)=x$ and $\gamma(1)=y$. On the one hand,

$$
k_{D}\left(x_{0}, x\right) \leqslant c_{3} m
$$

on

$$
D_{m}:=\bigcup\left\{Q \in \mathscr{W}: \frac{b^{-1}}{2^{m}} \leqslant \operatorname{diam}(Q) \leqslant \frac{b}{2^{m}}\right\}
$$

for every $m \geqslant 1$ and some constants $c_{3}, b>1$, where $\mathscr{W}=\{Q\}$ is a Whitney decomposition of $D$ into closed dyadic cubes with disjoint interiors, and $\operatorname{diam}(Q)$ denotes the diameter for a cube $Q \in \mathscr{W}$. Then, following the argument in [30, Page 76] of [30, Theorem 2], one can see that for each $\theta>0$,

$$
\int_{D} k_{D}^{\theta}\left(x_{0}, x\right) d x<\infty
$$


On the other hand, according to [30, Line 17 in Page 76], there is a constant $c_{4}>0$ such that for every $m \geqslant 1$

$$
\log \left(\frac{1}{\rho_{\partial D}(x)}\right) \leqslant c_{4} m, \quad x \in D_{m}
$$

From these, we can repeat the proof of [30, Theorem 2] and obtain that (1.7) holds any Hölder domain of order 0.

In the remainder of this subsection, we further assume that the Lévy process $X$ is symmetric, and adopt the following assumption on the Lévy measure $\nu$ :

(A2) For each $R>0$, there exist two constants $0<r_{1}<r_{2} \leqslant R$ such that

$$
S\left(r_{1}, r_{2}\right):=\left\{x \in \mathbb{R}^{d}: r_{1} \leqslant|x| \leqslant r_{2}\right\} \subseteq \operatorname{supp}(\nu) .
$$

It is obvious that (A2) is weaker than (A1).

For any $\theta, c, r>0$, define

$$
\beta_{\theta, c}(r)=4 \Phi_{0}\left(\frac{r}{2}\right) \Phi_{1}\left(e^{c\left(\Phi_{0}\left(\frac{r}{2}\right)\right)^{\frac{1}{\theta}}}\right),
$$

where

$$
\Phi_{0}(r)=(2 \pi)^{-d} \int e^{-r|q(\xi)|} d \xi, \quad \Phi_{1}(r)=\sup _{|\xi| \leqslant r}|q(\xi)| .
$$

We have the following statement for intrinsic ultracontractivity of $\left(T_{t}^{D}\right)_{t \geqslant 0}$ under (A2) and (LDI).

Theorem 1.2. Suppose that $X$ is a symmetric Lévy process such that (A2) holds true, and that (LDI) also holds for the bounded domain D. If there exists a constant $\theta>0$ such that for any $c>0$,

$$
\Psi_{\theta, c}(r):=\int_{r}^{\infty} \frac{\beta_{\theta, c}^{-1}(s)}{s} d s<\infty, \quad r \geqslant 1,
$$

then the associated Dirichlet semigroup $\left(T_{t}^{D}\right)_{t \geqslant 0}$ is intrinsically ultracontractive, and there are constants $c_{1}, c_{2}>0$ such that for all $t>0$ and $x, y \in D$,

$$
p^{D}(t, x, y) \leqslant c_{1} \Psi_{\theta, c_{2}}^{-1}(t \wedge 1) e^{-\lambda_{1} t} \phi_{1}(x) \phi_{1}(y) .
$$

Here, we use the convention that $f^{-1}(r)=\inf \{s>0: f(s) \leqslant r\}$ and $\inf \emptyset=\infty$.

The intrinsic ultracontractivity for Dirichlet semigroup of symmetric $\alpha$-stable process on a bounded Hölder domain of order 0 was established in [10]. Theorem 1.2 generalizes such result to more general symmetric Lévy process, whose Lévy measure may be singular or may not satisfy (A1). This can be seen from the following example.

Example 1.3. Let $X$ be a symmetric Lévy process with Lévy measure $\nu$ as follows

$$
\nu(A)=\sum_{i=0}^{\infty} \int_{A} \frac{1}{|z|^{d+\alpha}} \mathbb{1}_{\left\{2^{-2 i-1} \leqslant|z| \leqslant 2^{-2 i}\right\}} d z, \quad A \in \mathscr{B}\left(\mathbb{R}^{d}\right)
$$

for some $\alpha \in(0,2)$. Let $D$ be a bounded Hölder domain of order 0 . Then, the associated Dirichlet semigroup $\left(T_{t}^{D}\right)_{t \geqslant 0}$ is intrinsically ultracontractive, and for every $\theta>d / \alpha$, there exist constants $c_{1}, c_{2}>0$ such that

$$
p^{D}(t, x, y) \leqslant c_{1} e^{-\lambda_{1} t} \exp \left(c_{2}\left(1+t^{-\frac{d}{\alpha \theta-d}}\right)\right) \phi_{1}(x) \phi_{1}(y), \quad t>0, x, y \in D .
$$


The rest of this paper is arranged as follows. In Section 2 we present some preliminary results. Under assumptions (A1) and (RC), we verify that for general Lévy process the Dirichlet heat kernel $p^{D}(t, x, y)$ is strictly positive for every $t>0$ and $x, y \in D$. In particular, Corollary 2.4 here also yields the strictly positivity of the transition density $p(t, x, y)$, which is interesting of its own. In Section 3, we prove Theorem 1.1 by making use of the methods in [15, 21, 24] with some significant modifications. The last section is devoted to the proof of Theorem 1.2. Comparing with the idea used in Section 3, here we need establish the super Poincaré inequality for non-local Dirichlet forms and derive explicit lower bound for ground state in term of characteristic exponent.

\section{Preliminary Result: the Strict Positivity of Dirichlet Heat KERNEL}

The following lemma, similar to [15, Lemma 2.5], is a direct consequence of Assumption (A1).

Lemma 2.1. Suppose (A1) holds. Then for any $0<r<R_{0}$,

$$
\delta(r):=\inf _{|x| \leqslant R_{0}} \nu(B(x, r))>0,
$$

where $R_{0}>0$ is the constant in (A1).

Proof. Suppose that

$$
\inf _{|x| \leqslant R_{0}} \nu\left(B\left(x, r_{0}\right)\right)=0
$$

for some $0<r_{0}<R_{0}$. Then there exists a sequence $\left\{x_{n}\right\}_{n=0}^{\infty} \subseteq \overline{B\left(0, R_{0}\right)}$ such that

$$
\lim _{n \rightarrow \infty} x_{n}=x_{0}
$$

and

$$
\lim _{n \rightarrow \infty} \nu\left(B\left(x_{n}, r_{0}\right)\right)=0 .
$$

According to (2.10) and (1.5), for $n$ large enough

$$
\nu\left(B\left(x_{n}, r_{0}\right)\right) \geqslant \nu\left(B\left(x_{0}, \frac{r_{0}}{2}\right)\right)>0,
$$

which contradicts with (2.11). This proves our desired conclusion (2.9).

Next, we turn to the strictly positivity of the Dirichlet heat kernel $p^{D}(t, x, y)$. We first recall the parabolic property of the Dirichlet heat kernel $p^{D}(t, x, y)$ and the Lévy system of Lévy process $X$.

Lemma 2.2. (1) The Dirichlet heat kernel $p^{D}(t, x, y)$ enjoys the parabolic property, i.e. for any $0<s<t, x, y \in D$ and stopping time $\tau$ with $\tau \leqslant \tau_{D}$,

$$
p^{D}(t, x, y)=\mathbb{E}^{x}\left[p^{D}\left(t-\tau \wedge s, X_{\tau \wedge s}, y\right)\right] .
$$

(2) Let $f$ be a non-negative measurable function on $\mathbb{R}_{+} \times \mathbb{R}^{d} \times \mathbb{R}^{d}$ vanishing on the diagonal. Then for every $x \in \mathbb{R}^{d}$ and stopping time $T$,

$$
\mathbb{E}^{x}\left(\sum_{s \leqslant T} f\left(s, X_{s-}, X_{s}\right)\right)=\mathbb{E}^{x}\left[\int_{0}^{T} \int_{\mathbb{R}^{d}} f\left(s, X_{s}, X_{s}+z\right) \nu(d z) d s\right] .
$$


Proof. (1) We mainly follow the proof of [7, Theorem 4.5] to prove the parabolic property for $p^{D}(t, x, y)$. For fixed $t_{0}>0$ and $y \in D$, let $q(s, x)=p^{D}\left(t_{0}-s, x, y\right)$ on $\left[0, t_{0}\right) \times D$. For every $(t, x) \in\left[0, t_{0}\right) \times D$, define a $\mathbb{R}_{+} \times D$-valued process $Y$ by $Y_{s}:=\left(t+s, X_{s}^{D}\right)$ for $0 \leqslant s<t_{0}-t$, and denote $\left\{\widetilde{\mathscr{F}_{s}}, 0 \leqslant s<t_{0}-t\right\}$ by the associated natural filtration. The law of the space-time process $s \mapsto Y_{s}$ starting from $(t, x)$ will be denoted by $\mathbb{P}^{(t, x)}$. Since for each $t>0, \sup _{x, y \in D} p^{D}(t, x, y)<\infty$, $q\left(Y_{s}\right)$ is integrable for every $0 \leqslant s<t-t_{0}$. Then, for every $0<r<s<t_{0}-t$,

$$
\begin{aligned}
\mathbb{E}^{(t, x)}\left[q\left(Y_{s}\right) \mid \widetilde{\mathscr{F}_{r}}\right] & =\mathbb{E}^{x}\left[p^{D}\left(t_{0}-t-s, X_{s}^{D}, y\right) \mid \mathscr{F}_{r}\right] \\
& =\mathbb{E}^{X_{r}^{D}}\left[p^{D}\left(t_{0}-t-s, X_{s-r}^{D}, y\right)\right] \\
& =\int_{D} p^{D}\left(s-r, X_{r}^{D}, z\right) p^{D}\left(t_{0}-t-s, z, y\right) d z \\
& =p^{D}\left(t_{0}-t-r, X_{r}^{D}, y\right)=q\left(Y_{r}\right),
\end{aligned}
$$

where in the second equality $\left\{\mathscr{F}_{s}, 0 \leqslant s<t_{0}-t\right\}$ denotes the natural filtration generated by $X^{D}$ and we have used the Markov property of $X^{D}$, and the fourth equality follows from the semigroup property of the Dirichlet heat kernel. Hence, $\left\{q\left(Y_{s}\right), \widetilde{\mathscr{F}_{s}}, 0 \leqslant s<t_{0}-t\right\}$ is a martingale.

For every $t>0$, choosing $t_{0}=t$ in the definition of $q$ above and using the optional sampling theorem, we find for every $0<s<t$ and stopping time $\tau \leqslant \tau_{D}$

$$
p^{D}(t, x, y)=q(0, x)=\mathbb{E}^{(0, x)}\left[q\left(Y_{s \wedge \tau}\right)\right]=\mathbb{E}^{x}\left[p^{D}\left(t-s \wedge \tau, X_{s \wedge \tau}^{D}, y\right)\right] .
$$

This finishes the proof of (2.12).

(2) We can follow the argument of [8, Section 5] (in particular [8, (5.3)]) to get (2.13), and the details are omitted here.

The main result of this section is the following

Proposition 2.3. Let $X$ be a (not necessarily symmetric) Lévy process satisfying (A1), and let $D$ be an open (not necessarily bounded) set such that (RC) holds true. Then,

$$
p^{D}(t, x, y)>0, \quad \forall t>0, x, y \in D .
$$

As a direct consequence of Proposition 2.3, we have the following statement, which is interesting of its own.

Corollary 2.4. Let $X$ be a (not necessarily symmetric) Lévy process satisfying(A1). For any connected (not necessarily bounded) open set D,

$$
p^{D}(t, x, y)>0, \quad \forall t>0, x, y \in D .
$$

In particular,

$$
p(t, x, y)>0, \quad \forall t>0, x, y \in \mathbb{R}^{d},
$$

where $p(t, x, y)$ is the transition density for the process $X$.

Proof of Proposition 2.3. The proof is split into three steps, and the first two steps are devoted to the proof of Corollary 2.4.

(1) We show that for any connected open set $D, T_{t}^{D}\left(\mathbb{1}_{U}\right)(x)>0$ for every $x \in D$, connected open set $U \subseteq D$ and $t>0$. According to [3, Theorem 5.1], there is a 
constant $c_{0}>0$ such that for every $r, t>0$ and $x \in \mathbb{R}^{d}$

$$
\mathbb{P}^{x}\left(\tau_{B(x, r)}>t\right) \geqslant 1-c_{0} t \sup _{|\xi| \leqslant \frac{1}{r}}|q(\xi)| .
$$

In particular, for any $r>0$, we can find a constant $t(r)>0$ such that

$$
\mathbb{P}^{x}\left(\tau_{B(x, r)}>t(r)\right) \geqslant \frac{1}{2}, \quad \forall x \in \mathbb{R}^{d} .
$$

Let $R_{0}$ be the constant in Assumption (A1). Since $D$ is connected, for every $x \in D$, connected open set $U$ and $t>0$, there exist constants $\tilde{t}_{1}:=\tilde{t}_{1}(x, U, t)>0$, $0<\tilde{r}_{1}:=\tilde{r}_{1}(x, U, t)<\frac{R_{0}}{8}$ and a sequence $\left\{x_{i}\right\}_{i=1}^{N+1} \subseteq D$ with $N \geqslant\left[\frac{t}{\tilde{t}_{1}}\right] \geqslant 3$, such that the following properties hold:

(i) for every $1 \leqslant i \leqslant N, B_{i} \subseteq D, B_{N+1} \subseteq U$, and $B_{i} \bigcap B_{i+1}=\varnothing$, where $B_{i}:=B\left(x_{i}, 2 \tilde{r}_{1}\right)$ and $x_{1}=x$. (Note that we do not require that $B_{i} \bigcap B_{j}=\varnothing$ for any $i \neq j$, and so it may happen that $B_{i}=B_{j}$ for some $j \neq i+1$.)

(ii) for every $1 \leqslant i \leqslant N$ and $y_{i} \in B_{i},\left|y_{i}-y_{i+1}\right| \leqslant \frac{R_{0}}{2}$.

(iii) for every $z \in \mathbb{R}^{d}$,

$$
\mathbb{P}^{z}\left(\tau_{B\left(z, \tilde{r}_{1}\right)}>2 \tilde{t}_{1}\right) \geqslant \frac{1}{2} .
$$

Below, define a sequence of stopping times $\left\{\tilde{\tau}_{B_{i}}\right\}_{i=1}^{N}$ as follows

$$
\tilde{\tau}_{B_{0}}=0, \tilde{\tau}_{B_{1}}:=\tau_{B_{1}}, \tilde{\tau}_{B_{i+1}}:=\inf \left\{t>\tilde{\tau}_{B_{i}}: X_{t} \notin B_{i+1}\right\}, \quad 1 \leqslant i \leqslant N-1,
$$

and let $\tilde{B}_{i}:=B\left(x_{i}, \tilde{r}_{1}\right)$ for $1 \leqslant i \leqslant N+1$. Then, we have

$$
\begin{aligned}
& T_{t}^{D}\left(\mathbb{1}_{U}\right)(x) \\
& \geqslant T_{t}^{D}\left(\mathbb{1}_{B_{N+1}}\right)(x) \\
& =\mathbb{E}^{x}\left(\mathbb{1}_{B_{N+1}}\left(X_{t}^{D}\right)\right) \\
& \geqslant \mathbb{P}^{x}\left(\left(1-\frac{1}{N}\right) \tilde{t}_{1}<\tilde{\tau}_{B_{i}}-\tilde{\tau}_{B_{i-1}}<\tilde{t}_{1} \text { for each } 1 \leqslant i \leqslant N,\right. \\
& \text { and } \left.\forall \forall_{s \in\left[\tilde{\tau}_{B_{N}}, t\right]} X_{s}^{D} \in B_{N+1}\right) \\
& \geqslant \mathbb{P}^{x}\left(\left(1-\frac{1}{N}\right) \tilde{t}_{1}<\tilde{\tau}_{B_{i}}-\tilde{\tau}_{B_{i-1}}<\tilde{t}_{1} \text { and } X_{\tilde{\tau}_{B_{i}}} \in \tilde{B}_{i+1} \text { for each } 1 \leqslant i \leqslant N\right. \text {, } \\
& \text { and } \left.\forall_{s \in\left[\tilde{\tau}_{B_{N}}, t\right]} X_{s}^{D} \in B_{N+1}\right) \\
& =\mathbb{P}^{x}\left(\left(1-\frac{1}{N}\right) \tilde{t}_{1}<\tau_{B_{1}}<\tilde{t}_{1}, X_{\tau_{B_{1}}} \in \tilde{B}_{2} ;\right. \\
& \cdot \mathbb{P}^{X_{\tilde{\tau}_{B_{1}}}}\left(\left(1-\frac{1}{N}\right) \tilde{t}_{1}<\tau_{B_{2}}<\tilde{t}_{1}, X_{\tau_{B_{2}}} \in \tilde{B}_{3} ;\right. \\
& \cdot \mathbb{P}^{X_{\tilde{\tau}_{B}}}\left(\cdots \mathbb { P } ^ { X _ { \tilde { \tau } _ { B _ { N - 1 } } } } \left(\left(1-\frac{1}{N}\right) \tilde{t}_{1}<\tau_{B_{N}}<\tilde{t}_{1}, X_{\tau_{B_{N}}} \in \tilde{B}_{N+1}\right.\right. \text {; } \\
& \left.\left.\left.\left.\cdot \mathbb{P}^{X_{\tilde{\tau}_{B}}}\left(\forall_{s \in\left[0, t-\tilde{\tau}_{B_{N}}\right]} X_{s} \in B_{N+1}\right)\right) \cdots\right)\right)\right),
\end{aligned}
$$

where in the last equality we used the strong Markov property.

Note that, if for any $1 \leqslant i \leqslant N$,

$$
\left(1-\frac{1}{N}\right) \tilde{t}_{1}<\tilde{\tau}_{B_{i}}-\tilde{\tau}_{B_{i-1}}<\tilde{t}_{1}
$$


then

$$
t-\tilde{\tau}_{B_{N}} \leqslant t-N\left(1-\frac{1}{N}\right) \tilde{t}_{1}=t-N \tilde{t}_{1}+\tilde{t}_{1} \leqslant 2 \tilde{t}_{1}
$$

where the last inequality follows from the fact $t-N \tilde{t}_{1} \leqslant \tilde{t}_{1}$. Thus, when $X_{\tilde{\tau}_{B_{N}}} \in$ $\tilde{B}_{N+1}$ and $\left(1-\frac{1}{N}\right) \tilde{t}_{1}<\tilde{\tau}_{B_{i}}-\tilde{\tau}_{B_{i-1}}<\tilde{t}_{1}$ for all $1 \leqslant i \leqslant N$, we have

$$
\begin{aligned}
\mathbb{P}^{X_{\tilde{\tau}_{B}}}\left(\forall_{s \in\left[0, t-\tilde{\tau}_{B_{N}}\right]} X_{s} \in B_{N+1}\right) & \geqslant \inf _{y \in \tilde{B}_{N+1}} \mathbb{P}^{y}\left(X_{t} \in B\left(y, \tilde{r}_{1}\right) \text { for all } 0<t \leqslant 2 \tilde{t}_{1}\right) \\
& \geqslant \inf _{y \in \tilde{B}_{N+1}} \mathbb{P}^{y}\left(\tau_{B\left(y, \tilde{r}_{1}\right)}>2 \tilde{t}_{1}\right) \geqslant \frac{1}{2}
\end{aligned}
$$

where the last inequality we used (2.15).

On the other hand, for any $1 \leqslant i \leqslant N$, if $X_{\tilde{\tau}_{B_{i-1}}} \in \tilde{B}_{i}$, then, according to the Lévy system of the process $X$ (see Lemma 2.2),

$$
\begin{aligned}
& \mathbb{P}^{X_{\tilde{\tau}_{B_{i-1}}}}\left(\left(1-\frac{1}{N}\right) \tilde{t}_{1}<\tau_{B_{i}}<\tilde{t}_{1}, X_{\tau_{B_{i}}} \in \tilde{B}_{i+1}\right) \\
& \geqslant \inf _{y \in \tilde{B}_{i}} \int_{\left(1-\frac{1}{N}\right) \tilde{t}_{1}}^{\tilde{t}_{1}} \int_{B_{i}} p^{B_{i}}(s, y, z)\left(\int_{\tilde{B}_{i+1}-z} \nu(d w)\right) d z d s \\
& \geqslant \frac{\tilde{t}_{1}}{N}\left(\inf _{y \in \tilde{B}_{i}} \mathbb{P}^{y}\left(\tau_{B_{i}}>\tilde{t}_{1}\right)\right)\left(\inf _{z \in B_{i}} \nu\left(B\left(x_{i+1}-z, \tilde{r}_{1}\right)\right)\right) \\
& \geqslant \frac{\tilde{t}_{1}}{N}\left(\inf _{y \in \tilde{B}_{i}} \mathbb{P}^{y}\left(\tau_{B\left(y, \tilde{r}_{1}\right)}>\tilde{t}_{1}\right)\right)\left(\inf _{z \in B_{i}} \nu\left(B\left(x_{i+1}-z, \tilde{r}_{1}\right)\right)\right) .
\end{aligned}
$$

By (2.15),

$$
\inf _{y \in \tilde{B}_{i}} \mathbb{P}^{y}\left(\tau_{B\left(y, \tilde{r}_{1}\right)}>\tilde{t}_{1}\right)>\frac{1}{2}
$$

For every $z \in B_{i}$, since $\left|x_{i+1}-z\right| \leqslant \frac{R_{0}}{2}$ and $\tilde{r}_{1}<\frac{R_{0}}{2}, B\left(x_{i+1}-z, \tilde{r}_{1}\right) \subseteq B\left(0, R_{0}\right)$. Then, Assumption (A1) and Lemma 2.1 yield that

$$
\inf _{z \in B_{i}} \nu\left(B\left(x_{i+1}-z, \tilde{r}_{1}\right)\right)>0 .
$$

Therefore, for any $1 \leqslant i \leqslant N$ and $X_{\tilde{\tau}_{B_{i-1}}} \in \tilde{B}_{i}$,

$$
\mathbb{P}^{X_{\tilde{\tau}_{B_{i-1}}}}\left(\left(1-\frac{1}{N}\right) \tilde{t}_{1}<\tau_{B_{i}}<\tilde{t}_{1}, X_{\tau_{B_{i}}} \in \tilde{B}_{i+1}\right)>0 .
$$

Combining all the estimates above with (2.16), we obtain that $T_{t}^{D}\left(\mathbb{1}_{U}\right)(x)>0$.

(2) For any connected open set $D$, we have proved that $T_{t}^{D}\left(\mathbb{1}_{U}\right)(x)>0$ for any $x \in D, t>0$ and open connected subset $U \subseteq D$. So, $p^{D}(t, x, z)>0$ for almost surely $z \in D$ with respect to the Lebesgue measure (the exceptional set may depend on $x \in D$ and $t>0$ ). Furthermore, it is obvious that if Assumption (A1) holds for $\nu$, then it also holds for the Lévy measure $\hat{\nu}$ of the dual process $\hat{X}$. Then, following the arguments in step (1), we can obtain that for every $x \in D$ and $t>0, \hat{p}^{D}(t, x, z)>0$ for almost surely $z \in D$. 
Assume that $p^{D}(t, x, y)=0$ for some $x, y \in D$ and $t>0$. Then,

$$
\begin{aligned}
0=p^{D}(t, x, y) & =\int_{D} p^{D}\left(\frac{t}{2}, x, z\right) p^{D}\left(\frac{t}{2}, z, y\right) d z \\
& =\int_{D} p^{D}\left(\frac{t}{2}, x, z\right) \hat{p}^{D}\left(\frac{t}{2}, y, z\right) d z .
\end{aligned}
$$

On the other hand, according to the conclusions above, $p^{D}\left(\frac{t}{2}, x, z\right) \hat{p}^{D}\left(\frac{t}{2}, y, z\right)>0$ for almost surely $z \in D$, which is a contradiction with (2.17). Therefore, the assumption above is not true; that is, $p^{D}(t, x, y)>0$ for every $x, y \in D$ and $t>0$.

(3) Now we consider an open set satisfying (RC). It is easy to see that in this case for every $x, y \in D$, there exist an integer $m \geqslant 1$, some constants $0<\varepsilon<1$ and $0<r_{0}<\frac{\varepsilon R_{0}}{4}$ (here $R_{0}$ is the constant in $(\mathbf{A} 1)$ ) and points $x_{j} \in D$ for $1 \leqslant j \leqslant m$, such that

(i) $x \in B\left(x_{1}, r_{0}\right), y \in B\left(x_{m}, r_{0}\right)$.

(ii) for every $1 \leqslant j \leqslant m-1,\left|x_{j}-x_{j+1}\right| \leqslant(1-\varepsilon) R_{0}$.

(iii) for every $1 \leqslant i, j \leqslant m$ with $i \neq j, K_{i} \bigcap K_{j}=\varnothing$, where $K_{i}:=B\left(x_{i}, r_{0}\right)$.

For every $t>0,1 \leqslant j \leqslant m-1$ and $z_{j} \in K_{j}$, by the parabolic property of Dirichlet heat kernel $p^{D}(t, x, y)$ and the Lévy system of the process $X$, see Lemma 2.2,

$$
\begin{aligned}
& p^{D}\left(t, z_{j}, z_{j+1}\right) \\
& =\mathbb{E}^{z_{j}}\left[p^{D}\left(t-\frac{t}{2} \wedge \tau_{K_{j}}, X_{\frac{t}{2} \wedge \tau_{K_{j}}}, z_{j+1}\right)\right] \\
& \geqslant \mathbb{E}^{z_{j}}\left[p^{D}\left(t-\tau_{K_{j}}, X_{\tau_{K_{j}}}, z_{j+1}\right) \mathbb{1}_{\left\{\tau_{K_{j}} \leqslant \frac{t}{2}\right\}} \mathbb{1}_{\left\{X_{\tau_{K_{j}}} \in K_{j+1}\right\}}\right] \\
& =\int_{0}^{\frac{t}{2}} \int_{K_{j}} p^{K_{j}}\left(s, z_{j}, z\right) \int_{K_{j+1}-z} p^{D}\left(t-s, z+\tilde{z}, z_{j+1}\right) \nu(d \tilde{z}) d z d s \\
& \geqslant \int_{\frac{t}{4}}^{\frac{t}{2}} \int_{\tilde{K}_{j}} p^{K_{j}}\left(s, z_{j}, z\right) \int_{B\left(x_{j+1}-x_{j}, r_{0} / 2\right)} p^{D}\left(t-s, z+\tilde{z}, z_{j+1}\right) \nu(d \tilde{z}) d z d s,
\end{aligned}
$$

where the last inequality follows from the fact that $B\left(x_{j+1}-x_{j}, r_{0} / 2\right) \subseteq K_{j+1}-z$ for any $z \in \tilde{K}_{j}:=K_{j} / 2=B\left(x_{j}, r_{0} / 2\right)$.

By the conclusion in step (2), for every connected set $U \subseteq D$,

$$
p^{D}(t, x, y) \geqslant p^{U}(t, x, y)>0, \quad \forall t>0, x, y \in U
$$

According to (2.19) and the fact that for every $t>0, p^{D}(t, \cdot, \cdot): D \times D \rightarrow[0, \infty)$ is continuous, we know that

$$
\begin{aligned}
\inf _{z \in \tilde{K}_{j}, \tilde{z} \in B\left(x_{j+1}-x_{j}, r_{0} / 2\right)} & p^{D}\left(t-s, z+\tilde{z}, z_{j+1}\right) \\
& \geqslant \inf _{z \in \tilde{K}_{j}, \tilde{z} \in K_{j+1}-z} p^{D}\left(t-s, z+\tilde{z}, z_{j+1}\right) \\
& \geqslant \inf _{z \in K_{j+1}} p^{D}\left(t-s, z, z_{j+1}\right) \\
& =: C\left(t-s, r_{0}, x_{j+1}, z_{j+1}\right)>0 .
\end{aligned}
$$


Next, we suppose that $p^{D}\left(t, z_{j}, z_{j+1}\right)=0$ for some $t>0, z_{j} \in K_{j}$ and $1 \leqslant j \leqslant$ $m-1$. Then, by (2.18) and (2.20),

$$
\int_{\frac{t}{4}}^{\frac{t}{2}} C\left(t-s, r_{0}, x_{j+1}, z_{j+1}\right) \int_{\tilde{K}_{j}} p^{K_{j}}\left(s, z_{j}, z\right) \int_{B\left(x_{j+1}-x_{j}, r_{0} / 2\right)} \nu(d \tilde{z}) d z d s=0,
$$

which, along with (2.20), (A1) and the fact that $B\left(x_{j+1}-x_{j}, r_{0} / 2\right) \subseteq B\left(0, R_{0}\right)$ due to $\left|x_{j+1}-x_{j}\right| \leqslant(1-\varepsilon) R_{0}$ and $r_{0}<\frac{\varepsilon R_{0}}{4}$, in turn implies that

$$
p^{K_{j}}\left(s, z_{j}, z\right)=0
$$

holds for $(s, z) \in\left[\frac{t}{4}, \frac{t}{2}\right] \times \tilde{K}_{j}$ almost surely under the measure $d s d z$. However, according to (2.19), for every $s>0$ and $\tilde{x}, \tilde{y} \in K_{j}$

$$
p^{K_{j}}(s, \tilde{x}, \tilde{y})>0 .
$$

This is a contradiction with (2.21), whence

$$
p^{D}\left(t, z_{j}, z_{j+1}\right)>0, \quad \forall t>0, z_{j} \in K_{j}, 1 \leqslant j \leqslant m-1 .
$$

Finally, for every $t>0$ and $x, y \in D$,

$$
\begin{aligned}
p^{D}(t, x, y) & =\int_{D} \ldots \int_{D} p^{D}\left(\frac{t}{m}, x, z_{1}\right) p^{D}\left(\frac{t}{m}, z_{1}, z_{2}\right) \ldots p^{D}\left(\frac{t}{m}, z_{m}, y\right) d z_{1} \ldots d z_{m} \\
& \geqslant \int_{K_{1}} \ldots \int_{K_{m}} p^{D}\left(\frac{t}{m}, x, z_{1}\right) p^{D}\left(\frac{t}{m}, z_{1}, z_{2}\right) \ldots p^{D}\left(\frac{t}{m}, z_{m}, y\right) d z_{1} \ldots d z_{m} \\
& \geqslant \int_{K_{1}} \ldots \int_{K_{m}} p^{K_{1}}\left(\frac{t}{m}, x, z_{1}\right) p^{D}\left(\frac{t}{m}, z_{1}, z_{2}\right) \ldots \\
& \times p^{D}\left(\frac{t}{m}, z_{m-1}, z_{m}\right) p^{K_{m}}\left(\frac{t}{m}, z_{m}, y\right) d z_{1} \ldots d z_{m} .
\end{aligned}
$$

This along with (2.22) and (2.23) gives us that $p^{D}(t, x, y)>0$ for every $x, y \in D$ and $t>0$, which proves our desired assertion.

We conclude with two remarks on Proposition 2.3 and Corollary 2.4.

Remark 2.5. (1) When Lévy process $X$ is symmetric and $D$ is a bounded connected open set, the strict positivity of Dirichlet heat kernel $p^{D}(t, x, y)$ was proved in $[15$, Proposition 2.2(i)] without any additional condition on the Lévy measure. However, the proof heavily depends on the symmetric property, and it does not work for Corollary 2.4. An interesting point for Corollary 2.4 is due to that it is concerned about non-symmetric Lévy processes. Based on Proposition 2.3, some arguments for examples in [21, Section 4] can be shortened. Furthermore, according to the proofs of Proposition 2.3 and Lemma 4.3 below (in particular, see the construction of a sequence of subsets $\left\{D_{i}\right\}_{i=1}^{n}$ here), we can verify that, under the weaker assumption (A2) on the Lévy measure $\nu$, for any connected (not necessarily bounded) open set $D, p^{D}(t, x, y)>0$ for any $t>0$ and $x, y \in D$. The details are left to readers.

(2) The proof of Proposition 2.3 is only based on the probability estimate of the first exit time and the Lévy system of Lévy process $X$, both of which are available for general Lévy type processes, see e.g. [3, 8]. Therefore, Proposition 2.3 and so Corollary 2.4 still hold true for a large class of Lévy type jump processes. 


\section{Proof of Theorem 1.1}

Throughout this section, we always assume that assumption (A1) holds true, and the ground state $\phi_{1}$ and its dual ground state $\hat{\phi}_{1}$ are bounded, continuous and strictly positive. To prove Theorem 1.1, we mainly use the methods in $[15,21,24]$ but with non-trivial modifications. Since $D$ is a bounded set, there exist finite open subsets $\left\{\tilde{D}_{i}\right\}_{i=1}^{n}$ such that

(i) $D=\bigcup_{i=1}^{n} \tilde{D}_{i}$.

(ii) for any $1 \leqslant i \leqslant n$ and $\tilde{x}_{i}, \tilde{y}_{i} \in \tilde{D}_{i}$, we have $\left|\tilde{x}_{i}-\tilde{y}_{i}\right| \leqslant \frac{R_{0}}{2}$.

(iii) there are $0<r_{0}<\frac{R_{0}}{8}$ and finite points $\left\{x_{i}\right\}_{i=1}^{n}$ such that $B\left(x_{i}, 2 r_{0}\right) \subseteq$ $\overline{B\left(x_{i}, 2 r_{0}\right)} \subseteq \tilde{D}_{i}$ for every $1 \leqslant i \leqslant n$.

Below, we define

$$
A:=\bigcup_{i=1}^{n} B\left(x_{i}, \frac{r_{0}}{2}\right), \quad B:=\bigcup_{i=1}^{n} \overline{B\left(x_{i}, r_{0}\right)}, \quad C:=\bigcup_{i=1}^{n} B\left(x_{i}, 2 r_{0}\right) .
$$

For every open set $U \subseteq \mathbb{R}^{d}$, let

$$
G^{U}(x, y)=\int_{0}^{\infty} p^{U}(t, x, y) d t, \hat{G}^{U}(x, y)=\int_{0}^{\infty} \hat{p}^{U}(t, x, y) d t, \quad \forall x, y \in U
$$

be the Green functions for the Dirichlet semigroup $\left(T_{t}^{U}\right)_{t \geqslant 0}$ and $\left(\hat{T}_{t}^{U}\right)_{t \geqslant 0}$ respectively, e.g. see [21]. Define

$$
\eta_{U}=\inf \left\{t \geqslant 0, X_{t} \notin U\right\}, \quad \hat{\eta}_{U}=\inf \left\{t \geqslant 0, \hat{X}_{t} \notin U\right\} .
$$

We first provide the following estimate, which is crucial for the proof of Theorem 1.1 .

Lemma 3.1. There exists a constant $c_{1}>0$ such that for every $x \in \mathbb{R}^{d}$,

$$
\mathbb{P}^{x}\left(X_{\eta_{D \backslash B}} \in B\right) \geqslant c_{1} \mathbb{E}^{x}\left[\eta_{D \backslash B}\right], \quad \mathbb{P}^{x}\left(\hat{X}_{\eta_{D \backslash B}} \in B\right) \geqslant c_{1} \mathbb{E}^{x}\left[\hat{\eta}_{D \backslash B}\right] .
$$

Proof. For every $x \notin D$ or $x \in B$, we have $\mathbb{P}^{x}\left(\eta_{D \backslash B}=0\right)=1$, which immediately implies that the estimate for $X$ in (3.24) holds true. Now we assume $x \in D \backslash B$, and so $\eta_{D \backslash B}=\tau_{D \backslash B}, \mathbb{P}^{x}$-a.s.. By the Ikeda-Watanabe formula, see [15, (2.1)],

$$
\mathbb{P}^{x}\left(X_{\eta_{D \backslash B}} \in B\right) \geqslant \mathbb{P}^{x}\left(X_{\tau_{D \backslash B}} \in A\right)=\int_{D \backslash B} G^{D \backslash B}(x, y) \int_{A-y} \nu(d z) d y .
$$

For every $y \in D \backslash B$, there exists an integer $1 \leqslant i \leqslant n$ such that $y \in \tilde{D}_{i}$. Then, by the definition of $A$, we obtain

$$
B\left(x_{i}-y, \frac{r_{0}}{4}\right) \subseteq A-y .
$$

Moreover, since $y, x_{i} \in \tilde{D}_{i}$, by the property of $\tilde{D}_{i}$ we know that $\left|x_{i}-y\right| \leqslant \frac{R_{0}}{2}$. Combining (3.26) with (2.9) yields that for every $y \in D \backslash B$

$$
\nu(A-y) \geqslant \nu\left(B\left(x_{i}-y, \frac{r_{0}}{4}\right)\right) \geqslant \delta\left(\frac{r_{0}}{4}\right)>0 .
$$

According to (3.27) and (3.25),

$$
\mathbb{P}^{x}\left(X_{\eta_{D \backslash B}} \in B\right) \geqslant \delta\left(\frac{r_{0}}{4}\right) \int_{D \backslash B} G^{D \backslash B}(x, y) d y=\delta\left(\frac{r_{0}}{4}\right) \mathbb{E}^{x}\left[\tau_{D \backslash B}\right]=\delta\left(\frac{r_{0}}{4}\right) \mathbb{E}^{x}\left[\eta_{D \backslash B}\right],
$$

which arrived at the first desired assertion in (3.24) with $c_{1}=\delta\left(\frac{r_{0}}{4}\right)$. 
Following the arguments above, we can also obtain the estimate in (3.24) for the dual process $\hat{X}$.

Lemma 3.2. There exists a constant $c_{2}>0$ such that for every $x \in D$

$$
\begin{aligned}
& \int_{C} G^{D}(x, y) d y \geqslant c_{2} \int_{D \backslash C} G^{D}(x, y) d y \\
& \int_{C} \hat{G}^{D}(x, y) d y \geqslant c_{2} \int_{D \backslash C} \hat{G}^{D}(x, y) d y .
\end{aligned}
$$

Proof. The proof is mainly based on Lemma 3.1 and the argument of [21, Lemma 3.5] (see also [15, 24]). We present the sketch here for the sake of completeness. It suffices to show the first estimate in (3.28), since the second one for the dual process $\hat{X}$ can be proved similarly.

Let $\theta_{t}$ denote the $t$-time shift operator for the process $X$. Define a sequence of stopping times as follows

$$
S_{1}:=0, \quad T_{k}:=S_{k}+\eta_{D \backslash B} \circ \theta_{S_{k}}, \quad S_{k+1}:=T_{k}+\eta_{C} \circ \theta_{T_{k}}, \quad k \geqslant 1 .
$$

According to (3.24) and the strong Markov property, we immediately have that for every $x \in \mathbb{R}^{d}$ and $k \geqslant 1$,

$$
\mathbb{P}^{x}\left(X_{T_{k}} \in B\right) \geqslant c_{1} \mathbb{E}^{x}\left[T_{k}-S_{k}\right]
$$

By [21, Lemma 3.4],

$$
\lim _{k \rightarrow \infty} T_{k}=\lim _{k \rightarrow \infty} S_{k}=\tau_{D}, \quad \mathbb{P}^{x} \text { a.s.. }
$$

Therefore we have

$$
\begin{aligned}
\int_{C} G^{D}(x, y) d y & =\mathbb{E}^{x}\left[\int_{0}^{\tau_{D}} \mathbb{1}_{C}\left(X_{t}\right) d t\right] \\
& =\mathbb{E}^{x}\left[\sum_{k=1}^{\infty}\left(\int_{S_{k}}^{T_{k}} \mathbb{1}_{C}\left(X_{t}\right) d t+\int_{T_{k}}^{S_{k+1}} \mathbb{1}_{C}\left(X_{t}\right) d t\right)\right] \\
& \geqslant \mathbb{E}^{x}\left[\sum_{k=1}^{\infty} \int_{T_{k}}^{S_{k+1}} \mathbb{1}_{C}\left(X_{t}\right) d t\right] \\
& =\sum_{k=1}^{\infty} \mathbb{E}^{x}\left[S_{k+1}-T_{k}\right]
\end{aligned}
$$

where the first step follows from the relation $X_{t}=X_{t}^{D}$ for every $t<\tau_{D}$, and in the last step we have used the fact that $X_{t} \in C$ for every $T_{k}<t<S_{k+1}$.

It is well known that Lévy process enjoys the Feller property, i.e. its semigroup $T_{t}$ maps $C_{\infty}\left(\mathbb{R}^{d}\right)$ into $C_{\infty}\left(\mathbb{R}^{d}\right)$ for every $t>0$. By the separation property of Feller process,

$$
\inf _{y \in B} \mathbb{E}^{y} \tau_{C} \geqslant \frac{t_{0}}{2}
$$


for some constant $t_{0}>0$, see $[21,(3.2)]$. Hence, due to the strong Markov property again, for every $x \in \mathbb{R}^{d}$ and $k \geqslant 1$,

$$
\begin{aligned}
& \mathbb{E}^{x}\left[S_{k+1}-T_{k}\right]=\mathbb{E}^{x}\left[\mathbb{E}^{\left.X_{T_{k}}\left[\tau_{C}\right] ; T_{k}<\tau_{D}\right]}\right. \\
& \geqslant \mathbb{P}^{x}\left(X_{T_{k}} \in B\right) \inf _{y \in B} \mathbb{E}^{y} \tau_{C} \\
& \geqslant \frac{c_{1} t_{0}}{2} \mathbb{E}^{x}\left[T_{k}-S_{k}\right] \\
& =: c \mathbb{E}^{x}\left[T_{k}-S_{k}\right] \text {, }
\end{aligned}
$$

where the last inequality follows from (3.29). Combining this estimate with (3.31) yields that

$$
\begin{aligned}
\int_{C} G^{D}(x, y) d y & \geqslant c \sum_{k=1}^{\infty} \mathbb{E}^{x}\left[T_{k}-S_{k}\right] \\
& \geqslant c \mathbb{E}^{x}\left[\sum_{k=1}^{\infty} \int_{S_{k}}^{T_{k}} \mathbb{1}_{D \backslash C}\left(X_{t}\right) d t\right] \\
& =c \mathbb{E}^{x}\left[\sum_{k=1}^{\infty}\left(\int_{S_{k}}^{T_{k}} \mathbb{1}_{D \backslash C}\left(X_{t}\right) d t+\int_{T_{k}}^{S_{k+1}} \mathbb{1}_{D \backslash C}\left(X_{t}\right) d t\right)\right] \\
& =c \mathbb{E}^{x}\left[\int_{0}^{\tau_{D}} \mathbb{1}_{D \backslash C}\left(X_{t}\right) d t\right] \\
& =c \int_{D \backslash C} G^{D}(x, y) d y
\end{aligned}
$$

where in the forth step we have used again the fact that $X_{t} \in C$ for every $T_{k}<t<$ $S_{k+1}$. This proves the desired conclusion.

According to Lemma 3.2, we can give lower bound estimates for ground state $\phi_{1}$ and dual ground state $\hat{\phi}_{1}$.

Lemma 3.3. There exists a constant $c_{3}>0$, such that for every $x \in D$,

$$
\mathbb{E}^{x}\left[\tau_{D}\right] \leqslant c_{3} \phi_{1}(x), \quad \mathbb{E}^{x}\left[\hat{\tau}_{D}\right] \leqslant c_{3} \hat{\phi}_{1}(x) .
$$

Proof. We only verify the first estimate in (3.32) here. By (3.28) we have for every $x \in D$

$$
\mathbb{E}^{x}\left[\tau_{D}\right]=\int_{C} G^{D}(x, y) d y+\int_{D \backslash C} G^{D}(x, y) d y \leqslant\left(1+\frac{1}{c_{2}}\right) \int_{C} G^{D}(x, y) d y .
$$

Since $C$ is a precompact subset of $D$ and $\phi_{1}$ is strictly positive and continuous on $D$, there is a constant $C_{1}>0$ such that $\inf _{z \in C} \phi_{1}(z) \geqslant C_{1}$. Hence for every $x \in D$

$$
\begin{aligned}
\int_{C} G^{D}(x, y) d y & \leqslant \frac{1}{C_{1}} \int_{C} G^{D}(x, y) \phi_{1}(y) d y \\
& \leqslant \frac{1}{C_{1}} \int_{D} G^{D}(x, y) \phi_{1}(y) d y=\frac{1}{C_{1} \lambda_{1}} \phi_{1}(x),
\end{aligned}
$$

where in the equality we have used the fact that $\frac{\phi_{1}(x)}{\lambda_{1}}=\int_{D} G^{D}(x, y) \phi_{1}(y) d y$, see e.g. [9]. Combining this with (3.33), we arrive at the conclusion (3.32).

Now, we are in a position to present the 
Proof of Theorem 1.1. According to (3.32), for any $t>0, x, y \in D$,

$$
\begin{aligned}
p^{D}(t, x, y) & =\int_{D} p^{D}\left(\frac{t}{3}, x, z\right) \int_{D} p^{D}\left(\frac{t}{3}, z, w\right) p^{D}\left(\frac{t}{3}, w, y\right) d w d z \\
& \leqslant c\left(\frac{t}{3}\right)\left(\int_{D} p^{D}\left(\frac{t}{3}, x, z\right) d z\right)\left(\int_{D} \hat{p}^{D}\left(\frac{t}{3}, y, w\right) d w\right) \\
& =c\left(\frac{t}{3}\right) \mathbb{P}^{x}\left(\tau_{D}>\frac{t}{3}\right) \mathbb{P}^{y}\left(\hat{\tau}_{D}>\frac{t}{3}\right) \\
& \leqslant \frac{9 c\left(\frac{t}{3}\right)}{t^{2}} \mathbb{E}^{x}\left[\tau_{D}\right] \mathbb{E}^{y}\left[\hat{\tau}_{D}\right] \\
& \leqslant \frac{9 c_{3}^{2} c\left(\frac{t}{3}\right)}{t^{2}} \phi_{1}(x) \hat{\phi}_{1}(y)
\end{aligned}
$$

where in the first inequality we have used the facts that $p^{D}(t, w, y)=\hat{p}^{D}(t, y, w)$ and $\sup _{z, w \in D} p^{D}\left(\frac{t}{3}, z, w\right) \leqslant c\left(\frac{t}{3}\right)$, and the second inequality follows from the Chebyshev inequality. Hence, from (3.34) we know that the semigroup $\left(T_{t}^{D}\right)_{t \geqslant 0}$ is intrinsically ultracontractive.

Furthermore, according to [23], we know that for every $t>0$,

$$
c(t) \leqslant(2 \pi)^{-d} \int e^{-t \operatorname{Re} q(\xi)} d \xi,
$$

which together with (3.34) yields the desired assertion (1.6) for $t>0$ small enough. The estimate in (1.6) for large $t$ follows from [19, Theorem 2.7]. By now we have finished the proof.

To show the power of Theorem 1.1, we take the following example about the truncated strictly $\alpha$-stable process. In particular, comparing with [21, Example 4.5], we do not require that $D$ is $\kappa$-fat.

Example 3.4. Let $X$ be a Lévy process on $\mathbb{R}^{d}$ with Lévy measure as follows

$$
\nu(A) \geqslant c_{0} \int_{\mathbb{S}} \int_{0}^{r_{0}} \mathbb{1}_{A}(\theta r) \frac{1}{r^{d+\alpha}} d r \mu(d \theta), \quad A \in \mathscr{B}\left(\mathbb{R}^{d}\right),
$$

where $\alpha \in(0,2), r_{0}, c_{0}>0$ and $\mu$ is a finite non-degenerate (not necessarily symmetric) measure on the unit sphere $\mathbb{S}$ in the sense that its support is not contained in any proper linear subspace of $\mathbb{R}^{d}$. Let $D$ be a bounded open set satisfying assumption (RC) in Section 2 with $R_{0}$ to be the constant $r_{0}$ in (3.35). Then, the associated Dirichlet semigroup $\left(T_{t}^{D}\right)_{t \geqslant 0}$ is intrinsically ultracontractive, and for all $t>0$ and $x, y \in D$,

$$
p^{D}(t, x, y) \leqslant c_{1} e^{-\lambda_{1} t}\left(1+t^{-2-d / \alpha}\right) \phi_{1}(x) \hat{\phi}_{1}(y)
$$

holds for some constant $c_{1}>0$.

Proof. Let $\nu$ be the Lévy measure given by (3.35), and let $D$ be the open set satisfying (RC). According to Proposition 2.3, Corollary 2.4 and (the proof of) [29, Example 1.5], both the transition density $p(t, x, y)$ and the Dirichlet heat kernel $p^{D}(t, x, y)$ exist and fulfill all the conditions in Subsection 1.1. It is obvious that (A1) holds. Then, the desired assertion follows from Theorem 1.1.

Remark 3.5. (1) As mentioned in the beginning of Subsection 1.3, the intrinsic ultracontractivity of Dirichlet semigroups for Brownian motion on a bounded domain $D$ depends on the geometry of the boundary of $D$. Furthermore, by $[27$, Theorem 
1.1] and the conclusion of Example 3.4, quantitative estimates about $C(t)$ in (1.4) are also different for intrinsically contractive Dirichlet semigroups between Brownian motion and Lévy jump process on bounded Lipschitz domains.

(2) The conclusion (1.6) can apply to get explicit upper estimates for Dirichlt heat kernel $p^{D}(t, x, y)$. For instance, consider symmetric $\alpha$-stable process on bounded $\kappa$ fat domain $D$. Then, there is a constant $c>0$ such that for any $x, y \in D$ and $t \in(0,1]$,

$$
p^{D}(t, x, y) \leqslant c\left(t^{-d / \alpha} \wedge \frac{t}{|x-y|^{d+\alpha}}\right)\left(\frac{\phi_{1}(x)}{t} \wedge 1\right)\left(\frac{\phi_{1}(y)}{t} \wedge 1\right) .
$$

Indeed, it was shown in [2, Theorem 1] that for any $x, y \in D$ and $t \in(0,1]$,

$$
p^{D}(t, x, y) \asymp p(t, x, y) \mathbb{P}^{x}\left(\tau_{D}>t\right) \mathbb{P}^{y}\left(\tau_{D}>t\right),
$$

where $p(t, x, y)$ is the transition density of $\alpha$-symmetric stable process, i.e.

$$
p(t, x, y) \asymp\left(t^{-d / \alpha} \wedge \frac{t}{|x-y|^{d+\alpha}}\right) .
$$

On the other hand, according to (1.6), there is a constant $c_{1}>0$ such that

$$
p^{D}(t, x, y) \leqslant c_{1} t^{-d / \alpha-2} \phi_{1}(x) \phi_{1}(y), \quad x, y \in D, t \in(0,1] .
$$

By (3.36) and (3.37), we find that for some $c_{2}>0$,

$$
\mathbb{P}^{x}\left(\tau_{D}>t\right) \leqslant c_{2}\left(\frac{\phi_{1}(x)}{t} \wedge 1\right), \quad x \in D,
$$

which along with (3.36) in turn yields the desired assertion.

\section{Proof of Theorem 1.2}

The main tool to prove Theorem 1.2 is different from that of Theorem 1.1, and it is based on the (intrinsic) super Poincare inequality for non-local Dirichlet forms (this is the reason why we need require $X$ to be symmetric in the section). The super Poincaré inequality can be viewed as an alternative of Rosen's Lemma, which is in the context of the super log-Sobolev inequality, see e.g. [14, Theorem 5.1].

First, we recall some facts about Dirichlet form in our setting. Let $X$ be a symmetric Lévy process, and $D$ be a bounded domain. Then, the symmetric Dirichlet form $\left(\mathscr{E}^{D}, \mathscr{D}\left(\mathscr{E}^{D}\right)\right)$ for the Dirichlet semigroup $\left(T_{t}^{D}\right)_{t \geqslant 0}$ on $L^{2}(D ; d x)$ is given by

$$
\begin{aligned}
\mathscr{E}^{D}(f, f) & =\int_{\mathbb{R}^{d}} \int_{\mathbb{R}^{d}}(f(x+z)-f(x))^{2} \nu(d z) d x, \\
\mathscr{D}\left(\mathscr{E}^{D}\right) & ={\overline{C_{c}^{\infty}(D)}}^{\infty},
\end{aligned}
$$

where $C_{c}^{\infty}(D)$ is the set of $C^{\infty}$ functions on $D$ with compact support, and $\overline{C_{c}^{\infty}(D)}{ }^{\mathscr{E}_{1}^{D}}$ denotes the extension of $C_{c}^{\infty}(D)$ under the norm $\|f\|_{\mathscr{E}_{1} D}:=\sqrt{\mathscr{E}^{D}(f, f)+\|f\|_{L^{2}(D ; d x)}^{2}}$.

Since $D$ is connected, the Dirichlet heat kernel $p^{D}(t, x, y)$ is strictly positive for every $t>0$ and $x, y \in D$, e.g. see [15, Proposition 2.2(i)], and the associated ground state $\phi_{1}$ (corresponding to the first eigenvalue $\lambda_{1}$ ) can be chosen to be bounded, continuous and strictly positive. The following result is essentially taken from $[27$, Theorem 2.1 and Proposition 2.3], which give us sufficient conditions for intrinsic ultracontractivity of $\left(T_{t}^{D}\right)_{t \geqslant 0}$ in terms of (intrinsic) super Poincaré inequality for $\left(\mathscr{E}^{D}, \mathscr{D}\left(\mathscr{E}^{D}\right)\right)$ and lower bound of ground state for $\phi_{1}$. 
Lemma 4.1. Assume that there is a decreasing function $\beta_{0}:(0, \infty) \rightarrow(0, \infty)$ such that

$$
\int_{D} f^{2}(x) d x \leqslant s \mathscr{E}^{D}(f, f)+\beta_{0}(s)\left(\int_{D}|f(x)| d x\right)^{2}, \quad f \in C_{c}^{\infty}(D), s>0 .
$$

Then the following intrinsic super Poincaré inequality holds

$$
\int_{D} f^{2}(x) d x \leqslant s \mathscr{E}^{D}(f, f)+\beta(s)\left(\int_{D} \phi_{1}(x)|f(x)| d x\right)^{2}, \quad f \in C_{c}^{\infty}(D), s>0,
$$

where

$$
\beta(r)=\frac{4 \beta_{0}\left(\frac{r}{2}\right)}{\Theta\left(1 /\left(4 \beta_{0}\left(\frac{r}{2}\right)\right)\right)^{2}}, \Theta(r)=\sup \left\{s>0:\left|\left\{x \in D: \phi_{1}(x) \leqslant s\right\}\right| \leqslant r\right\} .
$$

If moreover

$$
\Psi(r)=\int_{r}^{\infty} \frac{\beta^{-1}(s)}{s} d s<\infty, \quad r \geqslant 1,
$$

then the associated Dirichlet semigroup $\left(T_{t}^{D}\right)_{t \geqslant 0}$ is intrinsically ultracontractive, and for some constant $c_{1}>0$,

$$
p^{D}(t, x, y) \leqslant c_{1} \Psi^{-1}(t \wedge 1) e^{-\lambda_{1} t} \phi_{1}(x) \phi_{1}(y),
$$

where $-\lambda_{1}<0$ is the eigenvalue associated with the ground state $\phi_{1}$.

According to Lemma 4.1, in order to prove Theorem 1.2 one only need to derive upper bound of $\beta_{0}(s)$ in the super Poincaré inequality (4.38), and lower bound of $\Theta(r)$ defined by (4.39). First, we have

Lemma 4.2. Let $X$ be a symmetric Lévy process given in Section 1.1. Then, the super Poincaré inequality (4.38) holds with

$$
\beta_{0}(r)=\Phi_{0}(r), \quad r>0,
$$

where $\Phi_{0}$ is given in (1.8).

Proof. By our assumption, the transition density $p(t, x, y)$ satisfies that

$$
\sup _{x, y \in \mathbb{R}^{d}} p(t, x, y) \leqslant c(t), \quad t>0 .
$$

As mentioned in the proof of Theorem 1.1,

$$
c(t) \leqslant(2 \pi)^{-d} \int e^{-t|q(\xi)|} d \xi=\Phi_{0}(t), \quad t>0,
$$

see e.g. [23]. Then, the desired assertion follows from the estimate above and [32, Theorem 3.3(2)] (or [33, Theorem 3.3.15]).

Next, we turn to lower bound estimate for the ground state, which seems to be interesting of itself.

Lemma 4.3. Let $X$ be a (not necessarily symmetric) Lévy process such that (A2) is satisfied, and let $D$ be a bounded domain. Then there is a constant $c_{1}>0$ such that

$$
\phi_{1}(x) \geqslant \frac{c_{1}}{\Phi_{1}\left(\frac{1}{\rho_{\partial D}(x)}\right)}, \quad x \in D
$$

where $\Phi_{1}$ is given in (1.8). 
If moreover (LDI) holds for $D$, then for any $\theta>0$, there exists constants $c_{2}, c_{3}>0$ such that

$$
\Theta(r) \geqslant \frac{c_{2}}{\Phi_{1}\left(e^{c_{3} r^{-\frac{1}{\theta}}}\right)}, \quad r>0 .
$$

Proof. (1) Since $\phi_{1}$ is continuous and strictly positive on $D$, it suffices to show (4.40) holds outside some compact subset of $D$. According to [3, Theorem 5.1], there is a constant $C_{1}>0$ such that for every $r>0, t>0$ and $x \in \mathbb{R}^{d}$

$$
\mathbb{P}^{x}\left(\tau_{B(x, r)}>t\right) \geqslant 1-C_{1} t \sup _{|\xi| \leqslant \frac{1}{r}}|q(\xi)|=1-C_{1} t \Phi_{1}\left(\frac{1}{r}\right) .
$$

Take $B\left(x_{0}, 2 r_{0}\right) \subseteq D$ with some $x_{0} \in D$ and $r_{0}>0$. According to (A2), there exist constants $0<r_{1}<r_{2} \leqslant \frac{r_{0}}{16}$ such that for every ball $B(z, r) \subseteq S\left(r_{1}, r_{2}\right)$, $\nu(B(z, r))>0$. Then, according to the proof of Lemma 2.1,

$$
\zeta\left(r, r_{1}, r_{2}\right):=1 \wedge \inf _{z \in \mathbb{R}^{d: B(z, r) \subseteq S\left(r_{1}, r_{2}\right)}} \nu(B(z, r))>0 .
$$

Below, we write $\zeta(r)$ for $\zeta\left(r, r_{1}, r_{2}\right)$, and let $\tilde{r}:=\frac{r_{1}+r_{2}}{2}$.

Since $D$ is bounded and connected, for every $y \in D$ with $\rho_{\partial D}(y) \leqslant \frac{\tilde{r}}{16}$, we can find finite points $\left\{y_{i}\right\}_{i=1}^{n}:=\left\{y_{i}(y)\right\}_{i=1}^{n} \subseteq D$ with some positive integer $n:=n(y)$, such that the following properties hold true:

(i) $y_{1}=y$.

(ii) $\left|y_{i+1}-y_{i}\right|=\tilde{r}$ for every $1 \leqslant i \leqslant n-1$.

(iii) There exists a constant $0<\varepsilon<\frac{1}{16} \wedge \frac{r_{2}-r_{1}}{3\left(r_{1}+r_{2}\right)}$ independent of $y$ such that $B\left(y_{i}, 2 \varepsilon \tilde{r}\right) \subseteq D$ for every $2 \leqslant i \leqslant n-1$, and $B\left(y_{n}, 2 \varepsilon \tilde{r}\right) \subseteq B\left(x_{0}, r_{0}\right)$.

(iv) There exists a positive integer $N$ such that

$$
\sup \left\{n(y): y \in D, \rho_{\partial D}(y) \leqslant \frac{\tilde{r}}{16}\right\} \leqslant N .
$$

In the following, for any $y \in D$ with $\rho_{\partial D}(y) \leqslant \frac{\tilde{r}}{16}$. Let $D_{1}:=B\left(y, \rho_{\partial D}(y)\right), \tilde{D}_{i}:=$ $B\left(y_{i}, \varepsilon \tilde{r}\right)$ and $D_{i}:=B\left(y_{i}, 2 \varepsilon \tilde{r}\right)$ for every $2 \leqslant i \leqslant n$. Define a sequence of stopping times as follows

$$
\tilde{\tau}_{D_{1}}:=\tau_{D_{1}}, \tilde{\tau}_{D_{i+1}}:=\inf \left\{t>\tilde{\tau}_{D_{i}}: X_{t} \notin D_{i+1}\right\}, \quad i \geqslant 1 .
$$

Set $t_{0}:=\frac{1}{4 C_{1} \Phi_{1}\left(\frac{1}{\varepsilon \tilde{r}}\right)}$ and $t_{1}(y):=\frac{1}{4 C_{1} \Phi_{1}\left(\frac{1}{\rho_{\partial D}(y)}\right)}$.

For any $y \in D$ with $\rho_{\partial D}(y) \leqslant \min \left\{\varepsilon, \frac{1}{16}\right\} \tilde{r}$, we have $t_{1}(y) \leqslant t_{0}$, and

$$
\begin{aligned}
& T_{2 t_{0}}^{D}\left(\mathbb{1}_{B\left(x_{0}, r_{0}\right)}\right)(y) \\
& \geqslant T_{2 t_{0}}^{D}\left(\mathbb{1}_{D_{n}}\right)(y) \\
& =\mathbb{E}^{y}\left(\mathbb{1}_{D_{n}}\left(X_{2 t_{0}}^{D}\right)\right) \\
& \geqslant \mathbb{P}^{y}\left(0<\tilde{\tau}_{D_{1}}<t_{1}(y), 0<\tilde{\tau}_{D_{i}}-\tilde{\tau}_{D_{i-1}}<\frac{t_{0}}{n},\right. \\
& \left.\quad X_{\tilde{\tau}_{D_{i-1}}}^{D} \in \tilde{D}_{i} \text { for each } 2 \leqslant i \leqslant n, \text { and } \forall_{s \in\left[\tilde{\tau}_{D_{n}}, 2 t_{0}\right]} X_{s}^{D} \in D_{n}\right) \\
& =\mathbb{P}^{y}\left(0<\tilde{\tau}_{D_{1}}<t_{1}(y), 0<\tilde{\tau}_{D_{i}}-\tilde{\tau}_{D_{i-1}}<\frac{t_{0}}{n},\right. \\
& \left.\quad X_{\tilde{\tau}_{D_{i-1}}} \in \tilde{D}_{i} \text { for each } 2 \leqslant i \leqslant n, \text { and } \forall_{s \in\left[\tilde{\tau}_{D_{n}}, 2 t_{0}\right]} X_{s} \in D_{n}\right)
\end{aligned}
$$




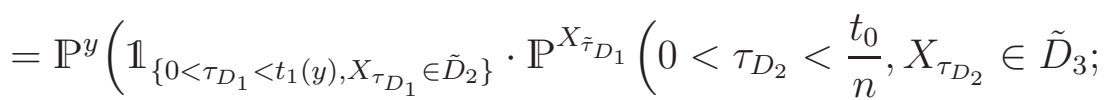

$$
\begin{aligned}
& \cdot \mathbb{P}^{X_{\tilde{\tau}_{D_{2}}}}\left(\cdots \mathbb { P } ^ { X _ { \tilde { \tau } _ { D _ { n - 2 } } } } \left(0<\tau_{D_{n-1}}<\frac{t_{0}}{n}, X_{\tau_{D_{n-1}}} \in \tilde{D}_{n} ;\right.\right.
\end{aligned}
$$

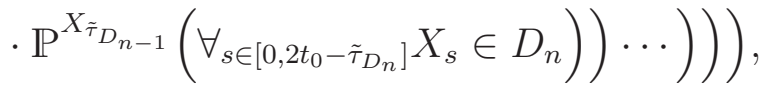

where the last equality follows from the strong Markov property.

By (4.41), for every $x \in D$,

$$
\mathbb{P}^{x}\left(X_{t} \in B(x, \varepsilon \tilde{r}) \text { for all } 0<t \leqslant 2 t_{0}\right) \geqslant \mathbb{P}^{x}\left(\tau_{B(x, \varepsilon \tilde{r})}>2 t_{0}\right) \geqslant \frac{1}{2},
$$

which gives us that

$$
\mathbb{P}^{X_{\tilde{\tau}_{D_{n-1}}}}\left(\forall_{s \in\left[0,2 t_{0}-\tilde{\tau}_{D_{n}}\right]} X_{s} \in D_{n}\right) \geqslant \inf _{x \in \tilde{D}_{n}} \mathbb{P}^{x}\left(X_{t} \in B(x, \varepsilon \tilde{r}) \text { for all } 0<t \leqslant 2 t_{0}\right) \geqslant \frac{1}{2} \text {. }
$$

On the other hand, for any $2 \leqslant i \leqslant n-1$, if $X_{\tilde{\tau}_{D_{i-1}}} \in \tilde{D}_{i}$, then, by the Lévy system of the process $X$, see Lemma 2.2,

$$
\begin{aligned}
& \mathbb{P}^{X_{\tilde{\tau}_{D_{i-1}}}}\left(0<\tau_{D_{i}}<\frac{t_{0}}{n}, X_{\tau_{D_{i}}} \in \tilde{D}_{i+1}\right) \\
& \geqslant \inf _{y \in \tilde{D}_{i}} \int_{0}^{\frac{t_{0}}{n}} \int_{D_{i}} p^{D_{i}}(s, y, z)\left(\int_{\tilde{D}_{i+1}-z} \nu(d w)\right) d z d s \\
& \geqslant \frac{t_{0}}{n}\left(\inf _{y \in \tilde{D}_{i}} \mathbb{P}^{y}\left(\tau_{D_{i}}>\frac{t_{0}}{n}\right)\right)\left(\inf _{z \in D_{i}} \nu\left(B\left(y_{i+1}-z, \varepsilon \tilde{r}\right)\right)\right) .
\end{aligned}
$$

For every $w \in B\left(y_{i+1}-z, \varepsilon \tilde{r}\right)$ and $z \in D_{i}$, it holds that

$$
\left|w-\left(y_{i+1}-y_{i}\right)\right| \leqslant\left|w-\left(y_{i+1}-z\right)\right|+\left|z-y_{i}\right| \leqslant 3 \varepsilon \tilde{r}
$$

and so

$$
r_{1} \leqslant\left|y_{i+1}-y_{i}\right|-3 \varepsilon \tilde{r} \leqslant|w| \leqslant\left|y_{i+1}-y_{i}\right|+3 \varepsilon \tilde{r} \leqslant r_{2}
$$

This implies that

$$
B\left(y_{i+1}-z, \varepsilon \tilde{r}\right) \subseteq S\left(r_{1}, r_{2}\right) .
$$

Using (4.42) and (4.43), we find that the right hand side of (4.44) is bigger than

$$
\frac{t_{0} \zeta(\varepsilon \tilde{r})}{N}\left(\inf _{y \in \tilde{D}_{i}} \mathbb{P}^{y}\left(\tau_{B(y, \varepsilon \tilde{r})}>t_{0}\right)\right) \geqslant \frac{3 t_{0} \zeta(\varepsilon \tilde{r})}{4 N} .
$$

Similarly, we can obtain that for every $y \in D$ with $\rho_{\partial D}(y) \leqslant \min \left\{\varepsilon, \frac{1}{16}\right\} \tilde{r}$,

$$
\begin{aligned}
\mathbb{P}^{y}\left(0<\tau_{D_{1}}<t_{1}(y), X_{\tau_{D_{1}}} \in \tilde{D}_{2}\right) & \geqslant t_{1}(y) \zeta(\varepsilon \tilde{r}) \mathbb{P}^{y}\left(\tau_{B\left(y, \rho_{\partial D}(y)\right)}>t_{1}(y)\right) \\
& \geqslant \frac{3 t_{1}(y) \zeta(\varepsilon \tilde{r})}{4} \\
& =\frac{3 \zeta(\varepsilon \tilde{r})}{16 C_{1} \Phi_{1}\left(\frac{1}{\rho_{\partial D}(y)}\right)} .
\end{aligned}
$$

Combining all the estimates above yields that for every $y \in D$ with $\rho_{\partial D}(y) \leqslant$ $\min \left\{\varepsilon, \frac{1}{16}\right\} \tilde{r}$,

$$
T_{2 t_{0}}^{D}\left(\mathbb{1}_{B\left(x_{0}, r_{0}\right)}\right)(y) \geqslant \frac{C_{2}\left(\frac{\zeta(\varepsilon \tilde{r})}{2 N}\right)^{N} \zeta(\varepsilon \tilde{r})}{\Phi_{1}\left(\frac{1}{\rho_{\partial D}(y)}\right)} \geqslant \frac{C_{3}}{\Phi_{1}\left(\frac{1}{\rho_{\partial D}(y)}\right)}
$$


Therefore, for every $y \in D$ with $\rho_{\partial D}(y) \leqslant \min \left\{\varepsilon, \frac{1}{16}\right\} \tilde{r}$

$$
\phi_{1}(y)=e^{2 \lambda_{1} t_{0}} T_{2 t_{0}}^{D}\left(\phi_{1}\right)(y) \geqslant e^{2 \lambda_{1} t_{0}}\left(\inf _{z \in B\left(x_{0}, r_{0}\right)} \phi_{1}(z)\right) T_{2 t_{0}}^{D}\left(\mathbb{1}_{B\left(x_{0}, r_{0}\right)}\right)(y) \geqslant \frac{C_{4}}{\Phi_{1}\left(\frac{1}{\rho_{\partial D}(y)}\right)},
$$

which proves (4.40).

(2) Suppose that $D$ satisfies (LDI). Then, (1.7) and the Chebyshev inequality yield that for each $\theta, s>0$,

$$
\begin{aligned}
\left|\left\{x \in D: \rho_{\partial D}(x) \leqslant s\right\}\right| & =\left|\left\{x \in D: \log \left(\frac{1}{\rho_{\partial D}(x)}\right) \geqslant c_{1} \log \frac{1}{s}\right\}\right| \\
& \leqslant \frac{\int_{D}\left|\log \left(\frac{1}{\rho_{\partial D}(x)}\right)\right|^{\theta} d x}{|\log s|^{\theta}} \leqslant \frac{C_{6}}{|\log s|^{\theta}} .
\end{aligned}
$$

Therefore, by (4.40) and the increasing of $\Phi_{1}(r)$, we get that for any $r, \theta>0$,

$$
\begin{aligned}
\Theta(r) & =\sup \left\{s>0:\left|\left\{x \in D: \phi_{1}(x)<s\right\}\right| \leqslant r\right\} \\
& \geqslant \sup \left\{s>0:\left|\left\{x \in D: \Phi_{1}\left(\frac{1}{\rho_{\partial D}(x)}\right) \geqslant \frac{c_{1}}{s}\right\}\right| \leqslant r\right\} \\
& \geqslant \sup \left\{s>0:\left|\left\{x \in D: \rho_{\partial D}(x) \leqslant\left[\Phi_{1}^{-1}\left(\frac{c_{1}}{s}\right)\right]^{-1}\right\}\right| \leqslant r\right\} \\
& \geqslant \sup \left\{s>0: \frac{C_{6}}{\log ^{\theta} \Phi_{1}^{-1}\left(\frac{c_{1}}{s}\right)} \leqslant r\right\} \\
& \geqslant \frac{C_{7}}{\Phi_{1}\left(e^{C_{8} r^{-\frac{1}{\theta}}}\right)},
\end{aligned}
$$

where $C_{7}, C_{8}$ are positive constants depending on $\theta$. The proof is complete.

Remark 4.4. According to [6, Theorem 1.1], (4.40) is not optimal for symmetric $\alpha$-stable process on bounded $C^{1,1}$-domain. However, as stated in Lemma 4.3, (4.40) holds for general (not necessarily symmetric) Lévy process and bounded domain with any regularity condition.

Proof of Theorem 1.2. Having Lemmas 4.1, 4.3 and 4.2 at hand, one can immediately obtain Theorem 1.2.

At the end of this section, we present the

Proof of Example 1.3. By the definition of Lévy measure $\nu$, it is clear that assumption (A2) holds.

By some element calculations, one can get that there is a constant $c_{2} \geqslant 1$ such that for $r>0$ small enough

$$
c_{2}^{-1} r^{-\alpha} \leqslant r^{-2} \int_{\{|z| \leqslant r\}}|z|^{2} \nu(d z) \leqslant \int\left(1 \wedge \frac{|z|}{r}\right)^{2} \nu(d z) \leqslant c_{2} r^{-\alpha} .
$$

According to [18, Proposition 1 and Lemma 5] and the inequality above, there exists a constant $c_{3} \geqslant 1$ such that for $r>0$ large enough,

$$
c_{3}^{-1} r^{\alpha} \leqslant \Phi_{1}(r) \leqslant c_{3} r^{\alpha},
$$

for $r>0$ small enough

$$
c_{3}^{-1} r^{-d / \alpha} \leqslant \Phi_{0}(r) \leqslant c_{3} r^{-d / \alpha} .
$$


Furthermore, on the one hand, by [23, Theorem 1], we know that the process $X$ has transition density $p(t, x, y)=p(t, 0, y-x)$ such that for each $t>0,(x, y) \mapsto$ $p(t, x, y): \mathbb{R}^{d} \times \mathbb{R}^{d} \rightarrow[0, \infty)$ is continuous. On the other hand, [18, Lemmas 5 and 7] and the Chapman-Kolmogorov equation for transition density $p(t, x, y)$ yield that for any $t>0$ and $x, y \in \mathbb{R}^{d}, p(t, x, y)>0$. Combining with all the conclusions above, we find that $p(t, x, y)$ satisfies all the assumptions in Subsection 1.1. Besides, it is easy to see that all the assumptions for $p^{D}(t, x, y)$ also hold true, thanks to [15, Proposition 2.2(i)].

The above estimates for $\Phi_{0}$ and $\Phi_{1}$ imply that for any $\theta>0$, there are constants $c_{4}, c_{5}>0$ such that for $r>0$ small enough,

$$
\beta(r) \leqslant c_{4} \exp \left(c_{5}\left(1+r^{-\frac{d}{\alpha \theta}}\right)\right) .
$$

Whence, the desired assertions follow from Theorem 1.2.

Acknowledgements. The authors are indebted to the referee for his/her careful corrections. The authors also would like to thank Professors Panki Kim, Renming Song and Feng-Yu Wang for their helpful comments on previous versions of the paper. Financial support through "Yang Fan Project" of Science and Technology Commission of Shanghai Municipality (No. 15YF1405900) (for Xin Chen), National Natural Science Foundation of China (No. 11201073), the JSPS postdoctoral fellowship (26-04021), National Science Foundation of Fujian Province (No. 2015J01003), and the Program for Nonlinear Analysis and Its Applications (No. IRTL1206) (for Jian Wang) is gratefully acknowledged.

\section{REFERENCES}

[1] Bertoin, J.: Lévy Processes, Cambridge Univ. Press, Cambridge 1996.

[2] Bogdan, K., Grzywny, T. and Ryznar, M.: Heat kernel estimates for the fractional Laplacian with Dirichlet conditions, Ann. Probab. 38 (2010), 1901-1923.

[3] Böttcher, B., Schilling, R.L. and Wang, J.: Lévy-Type Processes: Construction, Approximation and Sample Path Properties, Lecture Notes in Mathematics, vol. 2099, Lévy Matters III, Springer, Berlin 2014.

[4] Chen, X. and Wang, J.: Intrinsic contractivity of Feyman-Kac semigroups for symmetric jump processes with infinite range jumps, Front. Math. China 10 (2015), 753-776.

[5] Chen, X. and Wang, J.: Intrinsic contractivity of Feyman-Kac semigroups for symmetric jump processes, see arXiv:1403.3486

[6] Chen, Z.-Q., Kim, P. and Song, R.: Heat kernel estimates for Dirichlet fractional Laplacian, J. Euro. Math. Soc. 12 (2010), 1307-1329.

[7] Chen, Z.-Q. and Kumagai, T.: Heat kernel estimates for stable-like processes on $d$-sets, Stoch. Proc. Appl. 108 (2003), 27-62.

[8] Chen, Z.-Q. and Kumagai, T.: Heat kernel estimates for jump processes of mixed types on metric measure spaces, Probab. Theory Relat. Fields 140 (2008), 277-317.

[9] Chen, Z.-Q. and Song, R.: Intrinsic ultracontractivity and conditional gauge for symmetric stable processes, J. Funct. Anal. 150 (1997), 204-239.

[10] Chen, Z.-Q. and Song, R.: Intrinsic ultracontractivity, conditional lifetimes and conditional gauge for symmetric stable processes on rough domains, Illinois J. Math. 44 (2000), 138-160.

[11] Cipriani, F.: Intrinsic ultracontractivity of Dirichlet Laplacians in non-smooth domains, Potential Anal. 3 (1994), 203-218.

[12] Chung, K.L. and Zhao, Z.: From Brownian Motion to Schrödinger's Equation, SpringerVerlag, Berlin 1995.

[13] Davies, E.B.: Heat Kernels and Spectral Theory, Cambridge Univ. Press, Cambridge 1989.

[14] Davies, E.B. and Simon, B.: Ultracontractivity and heat kernels for Schrödinger operators and Dirichlet Laplacians, J. Funct. Anal. 59 (1984), 335-395. 
[15] Grzywny, T.: Intrinsic ultracontractivity for Lévy processes, Probab. Math. Statist. 28 (2008), 91-106.

[16] Kaleta, K and Kulczycki, T.: Intrinsic ultracontractivity for Schrödinger operators based on fractional Laplacians, Potential Anal. 33 (2010), 313-339.

[17] Kaleta, K. and Lőrinczi, J.: Pointwise eigenfunction estimates and intrinsic ultracontractivitytype properties of Feynman-Kac semigroups for a class of Lévy processes, Ann. Probab. 43 (2015), 1350-1398.

[18] Kaleta, K. and Sztonyk, P.: Estimates of transition densities and their derivatives for jump Lévy processes, J. Math. Anal. Appl. 431 (2015), 260-282.

[19] Kim, P. and Song, R.: Intrinsic ultracontractivity of non-symmetric diffusion semigroups in bounded domains, Tohoku Math. J. 60 (2008), 527-547.

[20] Kim, P. and Song, R.: Intrinsic ultracontractivity of non-symmetric diffusion semigroups with measure-valued drifts and potentials, Ann. Probab. 36 (2008), 1904-1945.

[21] Kim, P. and Song, R.: Intrinsic ultracontractivity for non-symmetric Lévy processes, Forum Math. 21 (2009), 43-66; Erratum, Forum Math. 21 (2009), 1137-1139.

[22] Knopova, V. and Schilling, R.L.: Transition density estimates for a class of Lévy and Lévytype processes, J. Theoret. Probab. 25 (2012), 144-170.

[23] Knopova, V. and Schilling, R.L.: A note on the existence of transition probability density for Lévy process, Forum Math. 25 (2013), 125-149.

[24] Kulczycki, T.: Intrinsic ultracontractivity for symmetric stable processes, Bull. Polish Acad. Sci. Math. 46 (1998), 325-334.

[25] Kulczycki, T. and Siddeja, B.: Intrinsic ultracontractivity of the Feynman-Kac semigroup for relativistic stable processes, Trans. Am. Math. Soc. 358 (2006), 5025-5057.

[26] Kwaśnicki, M.: Intrinsic ultracontractivity for stable semigroups on unbounded open sets, Potential Anal. 31 (2009), 57-77.

[27] Ouhabaz, E.-M. and Wang, F.-Y.: Sharp estimates for intrinsic ultracontractivity on $C^{1, \alpha_{-}}$ domains, Manuscripta Math. 122 (2007), 229-244.

[28] Schaefer, H.H.: Banach Lattices and Positive Operators, Springer-Verlag, New York 1974.

[29] Schilling, R., Sztonyk, P. and Wang, J.: Coupling property and gradient estimates for Lévy processes via the symbol, Bernoulli 18 (2012), 1128-1149.

[30] Smith, W. and Stegenga, D.: Hölder domains and Poincaré domains, Trans. Amer. Math. Soc. 319 (1990), 67-100.

[31] Sztonyk, P.: Transition density estimates for jump Lévy processes, Stochastic Process. Appl. 121 (2011), 1245-1265.

[32] Wang, F.-Y.: Functional inequalities and spectrum estimates: the infinite measure case, $J$. Funct. Anal. 194 (2002), 288-310.

[33] Wang, F.-Y.: Functional Inequalities, Markov Processes and Spectral Theory, Science Press, Beijing 2005. 